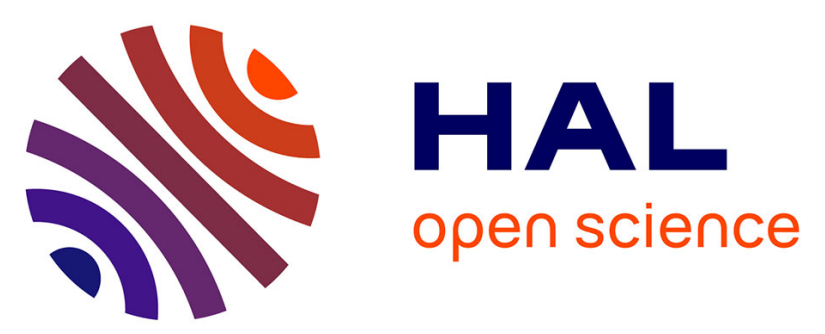

\title{
Finite-Time and Fixed-Time Observer Design: Implicit Lyapunov Function Approach
}

Francisco Lopez-Ramirez, Andrey Polyakov, Denis Efimov, Wilfrid

Perruquetti

\section{- To cite this version:}

Francisco Lopez-Ramirez, Andrey Polyakov, Denis Efimov, Wilfrid Perruquetti. Finite-Time and Fixed-Time Observer Design: Implicit Lyapunov Function Approach. Automatica, 2018, 87, pp.52 60. 10.1016/j.automatica.2017.09.007 . hal-01622356

\section{HAL Id: hal-01622356 \\ https://inria.hal.science/hal-01622356}

Submitted on 24 Oct 2017

HAL is a multi-disciplinary open access archive for the deposit and dissemination of scientific research documents, whether they are published or not. The documents may come from teaching and research institutions in France or abroad, or from public or private research centers.
L'archive ouverte pluridisciplinaire HAL, est destinée au dépôt et à la diffusion de documents scientifiques de niveau recherche, publiés ou non, émanant des établissements d'enseignement et de recherche français ou étrangers, des laboratoires publics ou privés. 


\title{
Finite-Time and Fixed-Time Observer Design: Implicit Lyapunov Function Approach
}

\author{
Francisco Lopez-Ramirez ${ }^{\mathrm{a}, *}{ }^{,}$Andrey Polyakov ${ }^{\mathrm{a}, \mathrm{b}}$, Denis Efimov $^{\mathrm{a}}$, \\ Wilfrid Perruquetti ${ }^{\text {a,b }}$
}

${ }^{a}$ Non-A INRIA - LNE, Parc Scientifique de la Haute Borne 40, avenue Halley Bat.A, Park Plaza 59650 Villeneuve d'Ascq, France

${ }^{\mathrm{b}}$ CRIStAL UMR CNRS 9189, Ecole Centrale de Lille, BP 48, Cité Scientifique, 59651 Villeneuve d'Ascq, France.

\begin{abstract}
This work deals with the problem of finite-time and fixed-time observation of linear multiple input multiple output (MIMO) control systems. The proposed nonlinear dynamic observers guarantee convergence of the observer states to the original system state in a finite and in a fixed (defined a priori) time. Algorithms for the observers parameters tuning are also provided and a robustness analysis against input disturbances and measurement noises is carried out. The theoretical results are illustrated by numerical examples that consider both noisy and noise-free measurements and a comparison with a high-gain observer is included.
\end{abstract}

Key words: Finite-Time Stability, Fixed-Time Stability, Implicit Lyapunov Function, Homogenous Systems

\section{Introduction}

State estimation (observation) is a classical problem in control theory [1], [2] that still forms an active research domain [3], [4], [5]. Observer design algorithms give background for the development of fault detection [6], data assimilation [7] systems and system parameter estimation [8], among other relevant research topics.

Observation with time constraints (non-asymptotic observation) is an interesting problem for both control theory [9] [10] and practice [11]. On the one hand, finite-time observation is a simple way to realize the separation principle, the condition under which the control and the observation algorithms can be designed and analyzed independently. On the other hand, in many control systems the transition processes are strongly restricted in time, e.g. the state estimate of a walking robot must be provided before each impact with the ground, i.e. calculated within the length of each step [12].

One of the most popular approaches to system state estimation is the so-called dynamic observer design, which uses a copy of the mathematical model of the system with an additional output injection term (see, for example, [2], [13], [14]

\footnotetext{
* Corresponding author. Tel. +33-359577800

Email addresses:

francisco.lopez-ramirezeinria.fr (Francisco

Lopez-Ramirez ), andrey.polyakoveinria.fr (Andrey

Polyakov), denis.efimov@inria.fr (Denis Efimov),

wilfrid.perruquetti@inria.fr(Wilfrid Perruquetti).
}

etc.). In this approach, the observer is designed by studying the stability of the error equation in order to guarantee finitetime or fixed-time (prescribed in advance) convergence of the observation error to zero. To attain this type of convergence, high order sliding mode (HOSM) differentiators [15], [16], [17] and homogeneous observers [11], [10], [3] can be used and they admit a rather simple and constructive representation. However, the practical implementation is complicated since, to the best of our knowledge, proper algorithms for parameter tuning and convergence time adjustment are not yet developed for high order systems.

The present paper, for which [18] is a preliminary version, develops effective computational algorithms for tuning the parameters of a finite-time observer and a novel fixed-time one; both of them based on homogeneity properties. In comparison with previous works, the algorithms here developed translate the observer parameter selection into a system of Linear Matrix Inequalities (LMI)[19], which allows to adjust the settling time.

Notation: Let $\lambda_{\min }(P)$ and $\lambda_{\max }(P)$ be the minimum and the maximum eigenvalues of the positive definite symmetric matrix $P \in \mathbb{R}^{n \times n}$, respectively; $I_{n} \in \mathbb{R}^{n \times n}$ denotes the identity matrix; $\operatorname{diag}\left(z_{1}, z_{2}, \ldots, z_{m}\right)$ denotes the diagonal matrix with diagonal elements $z_{i}, i=1,2, \ldots, m ; \operatorname{rown}(W)$ and $\operatorname{coln}(W)$ are the number of rows and the number of columns of the matrix $W$, respectively (e.g. if $W \in \mathbb{R}^{p \times q}$ then $\operatorname{rown}(W)=p$ and $\operatorname{coln}(W)=q) ; \operatorname{ker}(W)$ and range $(W)$ are the null space and the column space of the matrix $W$, respectively; $\operatorname{null}(W)$ is the matrix with columns 
defining the orthonormal basis of the subspace $\operatorname{ker}(W)$; $\mathbb{R}_{+}$denotes the set of nonnegative real numbers; $\mathbb{1}_{p}$ represents a vector of ones such that $\mathbb{1}_{p}=(1,1, \ldots, 1)^{T} \in \mathbb{R}^{p}$; $\mathcal{L}^{\infty}\left(\mathbb{R}^{p}\right)$ denotes the set of essentially bounded measurable functions $f: \mathbb{R}_{+} \rightarrow \mathbb{R}^{p}$ and the notation $\mathcal{L}_{(a, b)}^{\infty}$ is used for similar functions defined on $(a, b) ;|\cdot|$ denotes the absolute value in $\mathbb{R}$ and $\|\cdot\|$ denotes the Euclidean norm on $\mathbb{R}^{n}$.

\section{Problem statement}

Let us consider the perturbed linear control system:

$$
\left\{\begin{array}{l}
\dot{x}(t)=A x(t)+B u(t)+d_{x}(t), \\
y(t)=C x(t)+d_{y}(t),
\end{array}\right.
$$

where $x \in \mathbb{R}^{n}$ is the state variable, $y \in \mathbb{R}^{k}$ is the measured output, $u: \mathbb{R} \rightarrow \mathbb{R}^{s}$ is the control input, $A \in \mathbb{R}^{n \times n}$ is the system matrix, $B \in \mathbb{R}^{n \times s}$ is the matrix of input gains and the matrix $C \in \mathbb{R}^{k \times n}$ is the output matrix which links the measured outputs to the state variables. The pair $(A, C)$ is assumed to be observable and $\operatorname{rank}(C)=k$.

The goal of this work is twofold:

- Design two dynamic observers that estimate the state of the non-perturbed system (1) in a finite time or in a fixed (defined a priori) time, under the assumption that the domain of initial conditions is unknown.

- Both observers must be robust (in an input-to-state sense) with respect to $\mathcal{L}_{\infty}$-bounded measurement noises $d_{y}(t)$ and $\mathcal{L}_{\infty}$-bounded disturbances $d_{x}(t)$.

\section{Preliminaries}

\subsection{Finite-time and fixed-time stability}

Let us consider the system of the form

$$
\dot{x}(t)=f(t, x(t)), \quad t>t_{0}, \quad x\left(t_{0}\right)=x_{0},
$$

where $x \in \mathbb{R}^{n}$ is the state vector and $f: \mathbb{R}_{+} \times \mathbb{R}^{n} \rightarrow \mathbb{R}^{n}$ is a nonlinear continuous vector field. Let us assume that the origin is an equilibrium point of (2).

Definition 1 ([20], [21], [22]) The origin of system (2) is said to be globally uniformly finite-time stable if it is uniformly Lyapunov stable and finite-time attractive, i.e. there exists a locally bounded function $T: \mathbb{R}^{n} \rightarrow \mathbb{R}_{+} \cup\{0\}$ such that $x\left(t, t_{0}, x_{0}\right)=0$ for all $t \geq t_{0}+T\left(x_{0}\right)$, where $x\left(t, t_{0}, x_{0}\right)$ is a solution of (2) with $x_{0} \in \mathbb{R}^{n}$. The function $T$ is called the settling time function of the system (2).

Definition 2 ([23]) The origin of system (2) is said to be globally fixed-time stable if it is globally uniformly finitetime stable and the settling time function $T$ is globally bounded by some positive number $T_{\max }>0$, i.e. $T\left(x_{0}\right) \leq$ $T_{\max }, \forall x_{0} \in \mathbb{R}^{n}$.

A characterization of the fixed-time stability property by means of a Lyapunov function can be found in [10], [23].

\subsection{Implicit Lyapunov Function Method}

The Implicit Lyapunov Function (ILF) method allows to determine the stability of a system without presenting the Lyapunov function in an explicit form. Indeed, the Lyapunov function can remain in an implicit form, for example, as a solution of some algebraic equation (e.g. $Q(V, x)=0)$, and in order to analyze finite-time or fixed-time stability of a given system it is not necessary to solve this equation; it suffices to study some conditions involving the right-hand side of the system, as stated by the next theorem.

Theorem 3 ([24]) If there exists a continuous function $Q$ : $\mathbb{R}_{+} \times \mathbb{R}^{n} \rightarrow \mathbb{R}$ such that:

C1 $Q$ is continuously differentiable on $\mathbb{R}_{+} \times \mathbb{R}^{n} \backslash\{0\}$;

C2 for any $x \in \mathbb{R}^{n} \backslash\{0\}$ there exists $V \in \mathbb{R}_{+}: Q(V, x)=0$;

C3 for $\Omega=\left\{(V, x) \in \mathbb{R}^{n+1}: Q(V, x)=0\right\}$ we have

$$
\lim _{\substack{x \rightarrow 0 \\(V, x) \in \Omega}} V=0^{+}, \lim _{\substack{V \rightarrow 0^{+} \\(V, x) \in \Omega}}\|x\|=0, \lim _{\substack{\|x\| \rightarrow \infty \\(V, x) \in \Omega}} V=+\infty ;
$$

C4 $-\infty<\frac{\partial Q(V, x)}{\partial V}<0$ for $V \in \mathbb{R}_{+}$and for $x \in \mathbb{R}^{n} \backslash\{0\}$;

$$
\text { C5 } \frac{\partial Q(V, x)}{\partial x} f(t, x) \leq c V^{1-\mu} \frac{\partial Q(V, x)}{\partial V}, \forall t \in \mathbb{R}, \forall(V, x) \in \Omega \text {, }
$$

where $c>0$ and $0<\mu \leq 1$ are some constants. Then the origin of system (2) is uniformly finite-time stable with the settling time estimate $T\left(x_{0}\right) \leq \frac{V_{0}^{\mu}}{c \mu}$, where $V_{0} \in \mathbb{R}_{+}$: $Q\left(V_{0}, x_{0}\right)=0$.

The proof of this theorem is based on the classical Implicit Function Theorem [25] and recent results in the ILF method [26], [27], [24].

The next extension of the previous theorem allows to determine fixed-time stability using the ILF method.

Theorem 4 ([24]) Let two functions $Q_{1}(V, x)$ and $Q_{2}(V, x)$ satisfy the conditions C1-C4 of Theorem 3 and

C6 $Q_{1}(1, x)=Q_{2}(1, x), \forall x \in \mathbb{R}^{n} \backslash\{0\}$;

C7 $\begin{aligned} & \frac{\partial Q_{1}(V, x)}{\partial x} f(t, x) \leq c_{1} V^{1-\mu} \frac{\partial Q_{1}(V, x)}{\partial V}, \forall t \in \mathbb{R}, \\ & \forall V \in(0,1], \forall x \in\left\{z \in \mathbb{R}^{n} \backslash\{0\}: Q_{1}(V, z)=0\right\},\end{aligned}$ where $c_{1}>0$ and $0<\mu<1$ are some constants;

C8 $\frac{\partial Q_{2}(V, x)}{\partial x} f(t, x) \leq c_{2} V^{1+\nu} \frac{\partial Q_{2}(V, x)}{\partial V}, \forall t \in \mathbb{R}, \forall V \geq$ $1, \forall x \in\left\{z \in \mathbb{R}^{n} \backslash\{0\}: Q_{2}(V, z)=0\right\}$, where $c_{2}>0$ and $\nu>0$ are some constants.

Then the origin of (2) is fixed-time stable: $T\left(x_{0}\right) \leq \frac{1}{c_{1} \mu}+\frac{1}{c_{2} \nu}$.

The presented theorems are used below for analysis and design of finite-time and fixed-time observers. The corresponding implicit Lyapunov function candidate is selected as

$$
Q(V, z):=z^{T} D_{r}\left(V^{-1}\right) P D_{r}\left(V^{-1}\right) z-1
$$

where $V \in \mathbb{R}_{+}, z \in \mathbb{R}^{n}, P=P^{T} \in \mathbb{R}^{n \times n}$ is a positive definite matrix and $D_{r}(\cdot)$ is the dilation matrix of the form

$$
D_{r}(\lambda)=\operatorname{diag}\left(\lambda^{r_{1}} I_{n_{1}}, \lambda^{r_{2}} I_{n_{2}}, \ldots, \lambda^{r_{m}} I_{n_{m}}\right),
$$

with $r=\left(r_{1}, \ldots, r_{m}\right)^{T} \in \mathbb{R}^{m}, r_{i}>0, r_{\min }=\min _{1 \leq j \leq n} r_{j}$ and $n_{i}$ are natural numbers such that $n_{1}+\ldots+n_{m}=n$. Note that if $r_{1}=\ldots=r_{m}=0.5$ then $Q(V, z)=0$ implies that $V=z^{T} P z$. 


\subsection{Weighted Homogeneity and Input-to-State Stability}

The observers to be presented derive many of its properties from the fact that they exhibit a regular multiplicative scaling behavior, namely homogeneity. In this subsection we define this property and present some relevant theorems regarding robustness of homogeneous systems.

Consider the system in the form

$$
\dot{x}(t)=\tilde{f}(x(t), d(t)), \quad t>t_{0}, \quad x\left(t_{0}\right)=x_{0},
$$

where $x \in \mathbb{R}^{n}, d \in \mathcal{L}_{\infty}\left(\mathbb{R}^{p}\right)$ is a disturbance and $\tilde{f} \in$ $\mathbb{R}^{n+p} \rightarrow \mathbb{R}^{n}$ is a continuous or discontinuous vector field that satisfies Filippov conditions [28].

Definition 5 ([29]) A vector field $f: \mathbb{R}^{n} \rightarrow \mathbb{R}^{n}$ is $\boldsymbol{r}$ homogeneous with homogeneous weights $r \in \mathbb{R}^{n}$ and degree $\eta \in \mathbb{R}$, with $\eta \geq-r_{\min }$ if for all $x \in \mathbb{R}^{n}$ we have

$$
\lambda^{-\eta} D_{r}(\lambda)^{-1} f\left(D_{r}(\lambda) x\right)=f(x) .
$$

The system (5) for $d(t)=0$ is $r$-homogeneous of degree $\eta$ if the vector field $\tilde{f}(x(t), 0)$ is $r$-homogeneous of degree $\eta$.

Definition 6 ([29]) An r-homogeneous norm is a map $x \mapsto$ $\|x\|_{r, \tau}$, where for any $\tau \geq 0,\|x\|_{r, \tau}:=\left(\sum_{i=1}^{n}\left|x_{i}\right|^{\frac{\tau}{r_{i}}}\right)^{1 / \tau}$ $\forall x \in \mathbb{R}^{n}$. The set $S_{r}=\left\{x:\|x\|_{r, \tau}=1\right\}$ is the corresponding r-homogeneous unit sphere.

If a vector field fails to exhibit a global degree of homogeneity but behaves as an homogeneous vector field near infinity and/or near the origin, we say that it is locally homogeneous.

Definition 7 ([10], [30]) A vector field $f: \mathbb{R}^{n} \rightarrow \mathbb{R}^{n}$ is $\left(\boldsymbol{r}_{\mathbf{0}}, \boldsymbol{\lambda}_{\mathbf{0}}, \boldsymbol{f}_{\mathbf{0}}\right)$-homogeneous with degree $\eta_{0} \geq-r_{0 \min }\left(f_{0}\right.$ is an $r_{0}$-homogeneous vector field and $\left.r_{0 \min }=\min _{1 \leq j \leq n} r_{0 n}\right)$ and $\lambda_{0} \in \mathbb{R}_{+} \cup\{+\infty\}$ if $\lim _{\lambda \rightarrow \lambda_{0}}\left[\lambda^{-\eta_{0}} D_{r_{0}}^{-1}(\lambda) f\left(D_{r_{0}}(\lambda) x\right)-\right.$ $\left.f_{0}(x)\right]=0$, for all $x \in S_{r_{0}}$, uniformly on $S_{r_{0}}$ with $\lambda_{0} \in\{0, \infty\}$.

In the context of control and observation of nonlinear systems, the concept of input-to-state stability has won ground in characterizing the robustness of a system with respect to disturbances, noises and unmodeled dynamics since it allows to guarantee that for a given essentially bounded and measurable input, the system's state remains bounded [31],[32].

Definition 8 ([32]) The system (5) is called

Input-to-state stable (ISS), if there exist functions $\zeta \in \mathcal{K} \mathcal{L}$ and $\vartheta \in \mathcal{K}$ such that for any $d \in \mathcal{L}_{\infty}\left(\mathbb{R}^{p}\right)$ and any $x_{0} \in \mathbb{R}^{n}$ $\left\|x\left(t, t_{0}, d\right)\right\| \leq \zeta\left(\left\|x_{0}\right\|, t\right)+\vartheta\left(\|d\|_{[0, t)}\right), \forall t \geq 0$.

Integral intput-to-state stable (iISS), if there are some functions $\vartheta_{1}, \vartheta_{2} \in \mathcal{K}_{\infty}$ and $\zeta \in \mathcal{K} \mathcal{L}$ such that for any $x_{0} \in \mathbb{R}^{n}$ and $d \in \mathcal{L}_{\infty}\left(\mathbb{R}^{p}\right)$ the following estimate holds:

$$
\vartheta_{1}\left(\left\|x\left(t, x_{0}, d\right)\right\|\right) \leq \zeta\left(\left\|x_{0}\right\|, t\right)+\int_{0}^{t} \vartheta_{2}(\|d(s)\|) d s, \forall t \geq 0 .
$$

As the following theorem shows, homogeneity and homogeneous approximations allow to easily verify robustness properties, in an ISS sense, of a given system.
Theorem 9 ([30]) Let the vector field $\tilde{f}$ be homogeneous with weights $r=\left(r_{1}, \ldots, r_{n}\right)^{T}>0, \bar{r}=\left(\bar{r}_{1}, \ldots, \bar{r}_{p}\right)^{T} \geq$ 0 and with degree $\eta \geq-r_{\min }$, i.e. $\tilde{f}\left(D_{r}(\lambda) x, D_{\bar{r}}(\lambda) d\right)=$ $\lambda^{\eta} D_{r}(\lambda) \tilde{f}(x, d)$ for all $x \in \mathbb{R}^{n}, d \in \mathbb{R}^{p}$ and all $\lambda>0$. Assume that the system (5) is globally asymptotically stable for $d=0$, then the system (5) is

$$
\text { ISS if } \bar{r}_{\min }>0 \text { or iISS if } \bar{r}_{\min }=0 \text { and } \eta \leq 0 .
$$

In the case of locally homogeneous systems, ISS stability can be asserted through their homogeneous approximations at 0 and at $\infty$.

Theorem 10 ([10], Corollary 2.21) Let the vector field $\tilde{f}$ be continuous and $\left(\left(r^{\infty}, \bar{r}^{\infty}\right),+\infty, \tilde{f}_{\infty}\right)$-homogeneous with the weights $r^{\infty}=\left(r_{1}^{\infty}, \ldots, r_{n}^{\infty}\right)>0, \bar{r}^{\infty}=\left(\bar{r}_{1}^{\infty}, \ldots, \bar{r}_{p}^{\infty}\right)>$ 0 and $\left(\left(r^{0}, \bar{r}^{0}\right), 0, \tilde{f}_{0}\right)$-homogeneous with the weights $r^{0}=$ $\left(r_{1}^{0}, \ldots, r_{n}^{0}\right)>0, \bar{r}^{0}=\left(\bar{r}_{1}^{0}, \ldots, \bar{r}_{p}^{0}\right)>0$. If the origins of the systems $\dot{x}=\tilde{f}_{0}(x, 0), \dot{x}=\tilde{f}(x, 0)$ and $\dot{x}=\tilde{f}_{\infty}(x, 0)$ are globally asymptotically stable then the system (5) is ISS.

\section{Observer Design Using the ILF Method}

\subsection{Observer Canonical Decomposition}

The observers' design starts with a decomposition of the considered system into an appropriate block canonical form.

Lemma 11 Consider the system (1) with the pair $(A, C)$ being observable and $\operatorname{rank}(C)=k$. Then there exists a nonsingular transformation $\Phi$ such that

$$
\begin{aligned}
& \Phi A \Phi^{-1}=F \tilde{C}+\tilde{A}, C \Phi^{-1}=\left(\begin{array}{llll}
C_{0} & 0 & \ldots & 0
\end{array}\right), \tilde{C}=\left[\begin{array}{ll}
I_{k} & 0
\end{array}\right] \in \mathbb{R}^{k \times n}, \\
& \tilde{A}=\left(\begin{array}{ccccc}
0 & A_{12} & 0 & \ldots & 0 \\
0 & 0 & A_{23} & \ldots & 0 \\
\vdots & \vdots & \vdots & \ddots & \vdots \\
0 & 0 & 0 & \ldots & A_{m-1 m} \\
0 & 0 & 0 & \ldots & 0
\end{array}\right), C_{0} \in \mathbb{R}^{k \times k}, F \in \mathbb{R}^{n \times k},
\end{aligned}
$$

where $m$ is an integer, $A_{j-1 j} \in \mathbb{R}^{n_{j-1} \times n_{j}}, n_{j}=$ $\operatorname{rank}\left(A_{j-1 j}\right), j=2, \ldots, m$, so that $n_{1}=\operatorname{rank}(C)=k$ and $\sum_{i=1}^{m} n_{i}=n$.

We omit the proof of this lemma since it is a trivial consequence of well known results on block observability and controllability forms (see [33], [34], [35], [36]). However, in order to make the article self-contained we provide a suitable algorithm to calculate the transformation matrix $\Phi$ in the Appendix. If $k=1$ then $m=n, n_{i}=1$ and $\Phi$ transforms the matrix $A$ into the canonical Brunovsky form. It is also worth stressing that canonical forms and related transformations also exist for nonlinear systems (see for instance [37], [14]). Therefore, the observer design algorithms given below can be adapted to the nonlinear case.

\subsection{Finite-Time Observer}

Let us consider the following nonlinear observer

$$
\frac{d}{d t} \hat{x}(t)=A \hat{x}(t)+B u(t)-g_{F T}(y(t)-C \hat{x}(t)),
$$


where $\hat{x}(t) \in \mathbb{R}^{n}$ is the observer state vector and the function $g_{F T}: \mathbb{R}^{k} \rightarrow \mathbb{R}^{n}$ is defined as

$$
g_{F T}(\sigma)=\Phi^{-1}\left[D_{\tilde{r}}\left(\left\|\tilde{P} C_{0}^{-1} \sigma\right\|^{-1}\right) L_{F T}-F\right] C_{0}^{-1} \sigma,
$$

where $\sigma \in \mathbb{R}^{k}$, the matrices $\Phi \in \mathbb{R}^{n \times n}, C_{0} \in \mathbb{R}^{k \times k}$ and $F \in \mathbb{R}^{n \times k}$ are defined in Lemma $11, D_{\tilde{r}}(\cdot)$ is the dilation matrix given by (4) with

$$
\tilde{r}=\left(\frac{\mu}{1+(m-1) \mu}, \frac{2 \mu}{1+(m-1) \mu}, \ldots, \frac{m \mu}{1+(m-1) \mu}\right)^{T}, \mu \in(0,1],
$$

and $L_{F T} \in \mathbb{R}^{n \times k}$ and $\tilde{P} \in \mathbb{R}^{k \times k}$ are matrices of observer gains, to be determined. The error equation in the disturbance-free case (i.e. $d_{x}=0, d_{y}=0$ ) has the form

$$
\dot{e}=\left(\tilde{A}+D_{\tilde{r}}\left(\|\tilde{P} \tilde{C} e\|^{-1}\right) L_{F T} \tilde{C}\right) e,
$$

where $e=\Phi(x-\hat{x}), \tilde{A} \in \mathbb{R}^{n \times n}$ and $\tilde{C} \in \mathbb{R}^{k \times n}$ are defined in Lemma 11. Obviously, if $\mu \rightarrow 0$ then $D_{\tilde{r}}\left(\|\tilde{P} \tilde{C} e\|^{-1}\right) \rightarrow$ $I_{n}$ and the presented observer becomes the classical Luenberger one.

Remark 12 If the term $\left\|\tilde{P} C_{0}^{-1} \sigma\right\|$ in (7) is replaced with $\epsilon^{(1+(m-1) \mu) / \mu}$ where $\epsilon>0$ is a small constant, then the system (6)-(7) becomes a high-gain observer [38], [14] with the error dynamics given by $\dot{e}=\left(\tilde{A}+\operatorname{diag}\left(\epsilon^{-1} I_{n_{1}}, \epsilon^{-2} I_{n_{2}}, \cdots, \epsilon^{-m} I_{n_{m}}\right) L_{F T} \tilde{C}\right) e$. In our algorithms the gain factor $\epsilon$ depends on the available part of the observation error, namely, on $\sigma=y-C \hat{x}=C e$. This allows the finite-time and fixed-time observers to be less sensitive with respect to noises (see Section 6).

Let us define $H_{r}=\operatorname{diag}\left(r_{1} I_{n_{1}}, r_{2} I_{n_{2}}, \ldots, r_{m} I_{n_{m}}\right) \in \mathbb{R}^{n \times n}$, $r=\left[1+\frac{\mu}{1+(m-1) \mu}\right] \mathbb{1}_{m}-\tilde{r}$ and $\Xi(\lambda)=\lambda\left(D_{\tilde{r}}\left(\lambda^{-1}\right)-I_{n}\right)$.

Theorem 13 Let for some $\mu \in(0,1], \alpha>0, \xi>0$ and $\tau \geq 1$ the system of matrix inequalities

$$
\begin{aligned}
& \left(\begin{array}{cc}
P \tilde{A}+\tilde{A}^{T} P+\tilde{C}^{T} Y^{T}+Y \tilde{C}+\xi P+\alpha\left(P H_{r}+H_{r} P\right) & P \\
P & -\xi Z
\end{array}\right) \leq 0, \\
& P>0, Z>0, X>0, \text { (10b) } \quad\left(\begin{array}{cc}
\tau X & Y^{T} \\
Y & P
\end{array}\right) \geq 0, \\
& P H_{r}+H_{r} P>0, \quad(10 \mathrm{e}) \quad P \geq \tilde{C}^{T} X \tilde{C}, \\
& \Xi(\lambda) Z \Xi(\lambda) \leq \frac{1}{\tau} P, \quad \forall \lambda \in[0,1],
\end{aligned}
$$

be feasible for some $P, Z \in \mathbb{R}^{n \times n}, Y \in \mathbb{R}^{k \times n}$ and $X \in \mathbb{R}^{k \times k}$. Then the error equation (9) with $L_{F T}=P^{-1} Y$ and $\tilde{P}=X^{1 / 2}$ is globally finite-time stable with settling time $T \leq \frac{V^{\rho}(e(0))}{\alpha \rho}, \rho=\frac{\mu}{1+(m-1) \mu}$, where $V: \mathbb{R}^{n} \rightarrow \mathbb{R}$ is defined implicitly by the equation $Q(V, \cdot)=0$ with $Q$ given by (3).

In other words, this theorem claims that any solution of the observer system (6) converges to a solution of the real system (1) in a finite time $T$, which is dependent on the initial estimation error $e(0) \in \mathbb{R}^{n}$. The main idea of the proof is to show that the function $Q$ (defined in the statement of Theorem 13) satisfies all conditions of Theorem 3. Proofs of all theorems and propositions are given in the Appendix.

Corollary 14 The system of matrix inequalities (10) is feasible for sufficiently small $\mu>0$.

Indeed, observability of the pair $(A, C)$ implies that the pair $\left(\tilde{A}+0.5\left(\alpha H_{r}+\xi I_{n}\right), \tilde{C}\right)$ is also observable. Hence, it can be easily shown that the inequality (10a) is feasible with some positive definite matrices $P, Z \in \mathbb{R}^{n \times n}$ and $Y \in \mathbb{R}^{k \times n}$. The matrix inequalities (10d) and (10c) are also feasible for some $X \in \mathbb{R}^{k \times k}$ and sufficiently large $\tau \geq 1$. Since $\|\Xi(\lambda)\| \rightarrow 0$ uniformly on $\lambda \in[0,1]$ as $\mu \rightarrow 0$, then the inequalities (10e), (10f) will hold for sufficiently small $\mu>0$.

In order to apply Theorem 13 we need to solve the parametrized system of nonlinear matrix inequalities (10) with respect to variables $P, X, Z, Y$ for a given $\mu \in(0,1]$ and $\alpha, \xi, \tau>0$. By fixing the value $\lambda \in[0,1]$, the system (10) becomes a system of LMIs, which can be solved using any appropriate mathematical software (e.g. MATLAB). However, the mentioned LMIs must be checked for any $\lambda \in[0,1]$. Due to the smoothness of $\Xi(\lambda)$ with respect to $\lambda \in(0,1]$, this can be done on a proper grid constructed over this interval. The next corollary provides sufficient feasibility conditions for the parametrized matrix inequality (10f).

Proposition 15 The parametric inequality (10f) holds if

$$
\begin{gathered}
\Xi\left(q_{i}\right) Z \Xi\left(q_{i}\right)+\frac{1}{2}\left(q_{i}^{2}-q_{i-1}^{2}\right) M<\frac{1}{\tau} P, i=1, \ldots, N, \\
P>0, \quad Z>0, \quad M>0 \\
\left(\begin{array}{cc}
\left(I_{n}-H_{\tilde{r}}\right) Z+Z\left(I_{n}-H_{\tilde{r}}\right) & -Z H_{\tilde{r}} \\
-H_{\tilde{r}} Z & M
\end{array}\right)
\end{gathered}
$$

where $0=q_{0}<q_{1}<\ldots<q_{N}=1, H_{\tilde{r}}=\operatorname{diag}\left(\tilde{r}_{1} I_{n_{1}}\right.$, $\left.\tilde{r}_{2} I_{n_{2}}, \ldots, \tilde{r}_{m} I_{n_{m}}\right), P, M, Z \in \mathbb{R}^{n \times n}$.

The provided result allows the implementation of a simple algorithm to solve the parametrized system of matrix inequalities (10) with fixed $\alpha, \xi, \tau$ and $\mu$.

\section{Algorithm 1}

Initialization: $N=1, q_{0}=0, q_{N}=1, \Sigma=\left\{q_{0}, q_{N}\right\}$.

Loop: While the system of LMI (10a-10d), (11) is not feasible, do $\Sigma \leftarrow \Sigma \cup\left\{\frac{q_{i-1}+q_{i}}{2}\right\}_{i=1}^{N}$ and $N \leftarrow 2 N$.

Since the matrix inequality $\left(I_{n}-H_{\tilde{r}}\right) Z+Z\left(I_{n}-H_{\tilde{r}}\right)>0$ is obviously feasible for sufficiently small $\mu>0$, then, in the view of Corollary 14, the presented algorithm always finds the required solution if $\mu$ is sufficiently small.

\subsection{Fixed-Time Observer}

Let us consider now the following observer

$$
\frac{d}{d t} \hat{x}(t)=A \hat{x}+B u(t)-g_{F X}(y(t)-C \hat{x}(t)),
$$


where $\hat{x} \in \mathbb{R}^{n}$ and the function $g_{F X}: \mathbb{R}^{k} \rightarrow \mathbb{R}^{n}$ is defined as follows:

$g_{F X}(\sigma)=\Phi^{-1}\left[\frac{\left\{D_{\tilde{r}}\left(\left\|\tilde{P}_{1} C_{0}^{-1} \sigma\right\|^{-1}\right)+D_{\tilde{r}}\left(\left\|\tilde{P}_{2} C_{0}^{-1} \sigma\right\|\right)\right\} L_{F X}}{2}-F\right] C_{0}^{-1} \sigma$,

where $\sigma \in \mathbb{R}^{k}$, the matrices $\Phi \in \mathbb{R}^{n \times n}, C_{0} \in \mathbb{R}^{k \times k}$ and $F \in \mathbb{R}^{n \times k}$ are defined in Lemma 11. $\tilde{P}_{i} \in \mathbb{R}^{k \times k}, i=1,2$ and $L_{F X} \in \mathbb{R}^{n \times k}$ are gain matrices to be determined.

The error equation between (1) and (12) with $d_{x}=d_{y}=0$ is given by

$\dot{e}=\left(\tilde{A}+\frac{1}{2}\left\{D_{\tilde{r}}\left(\left\|\tilde{P}_{1} \tilde{C} e\right\|^{-1}\right)+D_{\tilde{r}}\left(\left\|\tilde{P}_{2} \tilde{C} e\right\|\right)\right\} L_{F X} \tilde{C}\right) e$,

$e=\Phi(x-\hat{x})$, where $\tilde{A} \in \mathbb{R}^{n \times n}$ and $\tilde{C} \in \mathbb{R}^{k \times n}$ are defined in Lemma 11.

Let us define $r_{i}=(-1)^{i} \tilde{r}+\left[1+\frac{(-1)^{i+1} \mu}{1+(m-1) \mu}\right] \mathbb{1}_{m}, \quad \bar{\Xi}_{i}^{\delta}(\lambda)=$ $\frac{\lambda_{1}}{2}\left\{D_{\tilde{r}}\left(\frac{\lambda_{2}^{i-1}}{\delta_{1} \lambda_{1}}\right)+D_{\tilde{r}}\left(\frac{\delta_{2} \lambda_{1}}{\lambda_{2}^{i-2}}\right)-2 I_{n}\right\}, \delta=\left(\delta_{1}, \delta_{2}\right), \lambda=$ $\left(\lambda_{1}, \lambda_{2}\right)$ and $H_{i}=\operatorname{diag}\left\{\left(r_{i}\right)_{1} I_{n_{1}}, \ldots,\left(r_{i}\right)_{m} I_{n_{m}}\right\} \in \mathbb{R}^{n \times n}$ for $i=1,2$.

Theorem 16 Let for some $\mu \in(0,1], \alpha>0, \xi>0, \tau \geq 1$ and $\delta=\left(\delta_{1}, \delta_{2}\right), \delta_{i}>0, i=1,2$, the system of matrix inequalities

$$
\begin{aligned}
& \left(\begin{array}{cc}
P \tilde{A}+\tilde{A}^{T} P+\tilde{C}^{T} Y^{T}+Y \tilde{C}+\xi P+\alpha\left(P H_{i}+H_{i} P\right) & P \\
P & -\xi Z_{i}
\end{array}\right) \leq 0, \\
& \left(\begin{array}{cc}
\tau X & Y^{T} \\
Y & P
\end{array}\right) \geq 0,(14 \mathrm{~b}) \quad P>0, X>0, Z_{i}>0, \\
& P H_{i}+H_{i} P>0,(14 \mathrm{~d}) \quad P \geq \tilde{C}^{T} X \tilde{C}, \\
& \bar{\Xi}_{i}^{\delta}(\lambda) Z_{i} \bar{\Xi}_{i}^{\delta}(\lambda) \leq \frac{1}{\tau} P, \quad \forall \lambda \in[0,1] \times[0,1],
\end{aligned}
$$

be feasible with $P, Z_{1}, Z_{2} \in \mathbb{R}^{n \times n}, Y \in \mathbb{R}^{n_{1} \times n}$ and $X \in$ $\mathbb{R}^{k \times k}$. Then the error equation (13) with $L_{F X}=P^{-1} Y, \tilde{P}_{i}=$ $\delta_{i} X^{1 / 2}$ is globally fixed-time stable with $T_{\max } \leq \frac{1+(m-1) \mu}{0.5 \alpha \mu}$.

Under the following additional restrictions, the parametric LMI (14f) can be simplified:

Proposition 17 Let $0=q_{0}<q_{1}<\ldots<q_{N_{1}}=1$ and $0<p_{0}<p_{1}<\ldots<p_{N_{2}}=1$ for some $N_{1}, N_{2} \geq 1$. If the positive definite matrices $Z_{i}, S_{i}, M_{i}, R_{i}, U_{i} \in \mathbb{R}^{n \times n}$ satisfy the following system of LMIs

$$
\begin{aligned}
& \left(\begin{array}{cc}
Z_{i} H_{\tilde{r}}+H_{\tilde{r}} Z_{i} & Z_{i} H_{\tilde{r}} \\
H_{\tilde{r}} Z_{i} & U_{i}
\end{array}\right) \geq 0, \quad(15 \mathrm{a}) \quad M_{i} H_{\tilde{r}}+H_{\tilde{r}} M_{i}>0, \\
& \left(\begin{array}{ccc}
2 Z_{i}-Z_{i} H_{\tilde{r}}-H_{\tilde{r}} Z_{i} & Z_{i} H_{\tilde{r}} & -Z_{i} H_{\tilde{r}} \\
H_{\tilde{r}} Z_{i} & M_{i} & 0 \\
-H_{\tilde{r}} Z_{i} & 0 & S_{i}
\end{array}\right) \geq 0, \\
& \bar{\Xi}_{i}^{\delta}\left(q_{j}, p_{s}\right) Z_{i} \bar{\Xi}_{i}^{\delta}\left(q_{j}, p_{s}\right)+ \\
& \ln \left(\frac{p_{s}}{p_{s-1}}\right) \bar{\Xi}_{i}^{\delta}\left(q_{j}, 0\right) U_{i} \bar{\Xi}_{i}^{\delta}\left(q_{j}, 0\right)+ \\
& \frac{q_{j}^{2}-q_{j-1}^{2}}{2}\left(D_{\tilde{r}}\left(\delta_{2}\right) M_{i} D_{\tilde{r}}\left(\delta_{2}\right)+S_{i}\right) \leq \frac{1}{\tau} P, \\
& \left(\begin{array}{cc}
\Upsilon_{i}\left(q_{j}, p_{0}\right) & \bar{\Xi}_{i}^{\delta}\left(q_{j}, 0\right) Z_{i} D_{\tilde{r}}\left(p_{0}^{1 / 2}\right) \\
D_{\tilde{r}}\left(p_{0}^{1 / 2}\right) Z_{i} \Xi_{i}^{\delta}\left(q_{j}, 0\right) & R_{i}
\end{array}\right) \geq 0,
\end{aligned}
$$

where $\Upsilon_{i}\left(q_{j}, p_{0}\right)=\frac{1}{\tau} P-\tilde{D}\left(q_{j}\right)\left(D_{\tilde{r}}\left(p_{0}\right) Z_{i} D_{\tilde{r}}\left(p_{0}\right)+\right.$ $\left.D_{\tilde{r}}\left(p_{0}^{1 / 2}\right) R_{i} D_{\tilde{r}}\left(p_{0}^{1 / 2}\right)\right) \tilde{D}\left(q_{j}\right)-\bar{\Xi}_{i}^{\delta}\left(q_{j}, 0\right) Z_{i} \bar{\Xi}_{i}^{\delta}\left(q_{j}, 0\right) \quad-$ $\frac{q_{j}^{2}-q_{j-1}^{2}}{2}\left(M_{i}+S_{i}\right)$ and $\tilde{D}_{i}\left(q_{j}\right):=\frac{q_{j}}{2} D_{\tilde{r}}\left(\left(\delta_{3-i} q_{j}\right)^{(-1)^{i}}\right)$, $i=1,2, j=1, \ldots, N_{1}, s=1, \ldots, N_{2}$, then (14f) holds.

Based on this proposition, an algorithm for solving the parametrized system of LMIs (14) can be presented, analogously with the finite-time case.

\section{Algorithm 2}

Initialization: $\beta>0, N_{1}=1, N_{2}=1, p_{0}>0, p_{N_{1}}=1$, $q_{0}=0, q_{N_{2}}=1, \Sigma=\left\{q_{0}, q_{N_{1}}\right\}, \Lambda=\left\{p_{0}, p_{N_{2}}\right\}$.

Loop: While the system of LMIs (14a-14e), (15) with $q_{j} \in \Sigma$, $p_{s} \in \Lambda$ is not feasible, do

$\Sigma \leftarrow \Sigma \cup\left\{0.5\left(q_{j-1}+q_{j}\right)\right\}_{j=1}^{N_{1}}$ with $q_{j} \in \Sigma, \quad N_{1} \leftarrow 2 N_{1}$,

$\Lambda \leftarrow \Lambda \cup\left\{p_{1} e^{-\beta}\right\}$ with $p_{1} \in \Lambda, \quad N_{2} \leftarrow N_{2}+1$.

Remark that the grid $\Lambda$ is a logarithmic grid such that the term $\ln \left(\frac{p_{s}}{p_{s-1}}\right)$ in (15d) equals $\beta$ for any $p_{s} \in \Lambda \backslash\left\{p_{0}, p_{1}\right\}$.

\section{Robustness Analysis}

We consider now (1) with nonzero $d_{x}: \mathbb{R}_{+} \rightarrow \mathcal{L}_{\infty}\left(\mathbb{R}^{n}\right)$, and nonzero $d_{y}: \mathbb{R}_{+} \rightarrow \mathcal{L}_{\infty}\left(\mathbb{R}^{k}\right)$. Since the observers' robustness follows from their homogeneity properties, we will establish, for each observer, the type of homogeneity that it exhibits and next that they are robust against bounded disturbances and bounded measurement noise. Again, the error variable is defined as $e=\Phi(x-\hat{x})$.

Corollary 18 Consider the perturbed error equation between (1) and (6)

$$
\dot{e}=\tilde{A} e+D_{\tilde{r}}\left(\left\|\tilde{P} \tilde{C} e+d_{y}\right\|^{-1}\right) L_{F T}\left(\tilde{C} e+d_{y}\right)+\phi,
$$

where $\phi=-F \tilde{C} d_{y}+\Phi d_{x}, d_{x} \in \mathcal{L}_{\infty}\left(\mathbb{R}^{n}\right), d_{y} \in \mathcal{L}^{\infty}\left(\mathbb{R}^{k}\right)$ and assume that all conditions of Theorem 13 are satisfied. Then the system (16) is ISS for $\mu \in(0,1)$ and iISS for $\mu=1$.

A similar result can be provided for the fixed-time observer:

Corollary 19 Consider the following perturbed error equation between (1) and (12)

$$
\dot{e}=\tilde{A} e+\frac{1}{2}\left\{D_{\tilde{r}}\left(\epsilon_{1}^{-1}\right)+D_{\tilde{r}}\left(\epsilon_{2}\right)\right\} L_{F X}\left(\tilde{C} e+d_{y}\right)+\phi,
$$

where $\phi=\Phi d_{x}-F \tilde{C} d_{y}, \epsilon_{i}=\left\|\tilde{P}_{i} \tilde{C} e+d_{y}\right\|, i=1,2, d_{x}, d_{y} \in \mathcal{L}^{\infty}$ and assume that all conditions of Theorem 16 are satisfied. Then the error dynamics (17) is ISS stable with respect to additive disturbances $d_{x}$ and measurement noises $d_{y}$.

Only qualitative analysis of robustness (i.e. ISS) is presented in this section. The quantitative one needs further research developments using ideas introduced in [39], [38], [40]. The LMIs presented in the previous section are expected to be useful for obtaining of rather precise ellipsoidal estimates of the observation error in the perturbed case.

\section{Examples}

The following two numerical simulations aim to show the main properties of the finite-time (FT) and the fixed-time 
(FX) observers. In both examples, an inverted-cart pendulum model will be used. In the first example, the robustness of the FT observer will be studied by applying it to the nonlinear plant and a comparison with a high-gain (HG) observer will be included. The second example will focus on the uniformity w.r.t to initial conditions (IC) of the FX observer. A linearized model will be used such that we can compare the performance of the observers with IC far away from the linearizing equilibrium point.

The state vector is given by $\mathbf{x}=[x, \dot{x}, \theta, \dot{\theta}]^{T}$, where as usual $(x, \dot{x})$ represents the position and the velocity of the cart and $(\theta, \dot{\theta})$ the angle (from the vertical down position) and the angular velocity of the pendulum. The model parameters are $M=0.5 \mathrm{Kg}$ - mass of the cart, $m_{c}=0.2 \mathrm{Kg}$ - mass of the pendulum, $b=0.1 \mathrm{~N} / \mathrm{m} / \mathrm{s}$ - cart friction coefficient, $l=0.3 \mathrm{~m}$ - length to pendulum center of mass, $I=0.006$ $\mathrm{Kg}$ - moment of inertia of the pendulum.

It is assumed that only position and angle can be measured directly. The nonlinear equations describing the system motion are given by

$$
\begin{aligned}
\left(M+m_{c}\right) \ddot{x}+b \dot{x}+m_{c}-l \ddot{\theta} \cos \theta-m_{c} l \dot{\theta}^{2} \sin \theta & =F_{\text {in }} \\
\left(I+m_{c} l^{2}\right) \ddot{\theta}+m_{c} g l \sin \theta+m_{c} l \ddot{x} \cos \theta & =0,
\end{aligned}
$$

where $F_{\text {in }}$ represents the input force. A simple proportional control law $F_{\text {in }}=K_{p} \frac{d}{d t} \hat{\theta}(t)$ is used to stabilize the pendulum around the downward position, $\frac{d}{d t} \hat{\theta}(t)$ is an estimate of the angular velocity to be obtained using the observers. The linearization of the model around the downward equilibrium point $\mathbf{x}_{0}=0 \in \mathbb{R}^{4}$ gives the following parameters for (1):

$$
A=\left(\begin{array}{cccc}
0 & 1 & 0 & 0 \\
0 & -0.1818 & 2.672 & 0 \\
0 & 0 & 0 & 1 \\
0 & 0.4545 & -31.181 & 0
\end{array}\right), B=\left(\begin{array}{c}
0 \\
1.818 \\
0 \\
-4.545
\end{array}\right), C=\left(\begin{array}{llll}
1 & 0 & 0 & 0 \\
0 & 0 & 1 & 0
\end{array}\right) \text {. }
$$

The first step of the observer design is to transform the linearized model into the observable canonical form, given in Lemma 11, by obtaining the matrix $\Phi=\left(\begin{array}{cccc}0 & 0 & 1 & 0 \\ -1 & 0 & 0 & 0 \\ a & -1 & 0 & 0 \\ b & 0 & 0 & 1\end{array}\right)$, where $a=-0.1818$ and $b=-0.4545$.

Example 1 (Comparison between FT and HG observers). In order to make a fair comparison we adjusted the parameters of both observers to have similar time response for the initial condition $x_{0}=(0,-2, \pi / 4,1)$; that is to say, the norm of the estimation errors is admitted to be less than 0.15 for $t \geq 0.5$ s. Using Algorithm 1 with $(\mu, \alpha, \tau, \xi)=(0.25,2.5,100,4)$ we design the FT observer (6), (7) with

$L_{F T}=\left(\begin{array}{cccc}-9.9080 & 0 & 0 & 0 \\ 0 & -9.9080 & -46.8452 & -46.8452 \\ 0\end{array}\right)^{T}, \tilde{P}=0.0969\left(\begin{array}{ll}1 & 0 \\ 0 & 1\end{array}\right)$,

and we compare it with the HG observer, designed according to Remark 12. Namely, the term $\left\|\tilde{P} C_{0}^{-1} \sigma\right\|$ in (7) is replaced with $\epsilon^{(1+(m-1) \mu) / \mu}$.

Note that, the gain factor $\epsilon=0.3$ (and correspondingly the admissible estimation error $\|e(0.5)\| \leq 0.15)$ is selected suf-
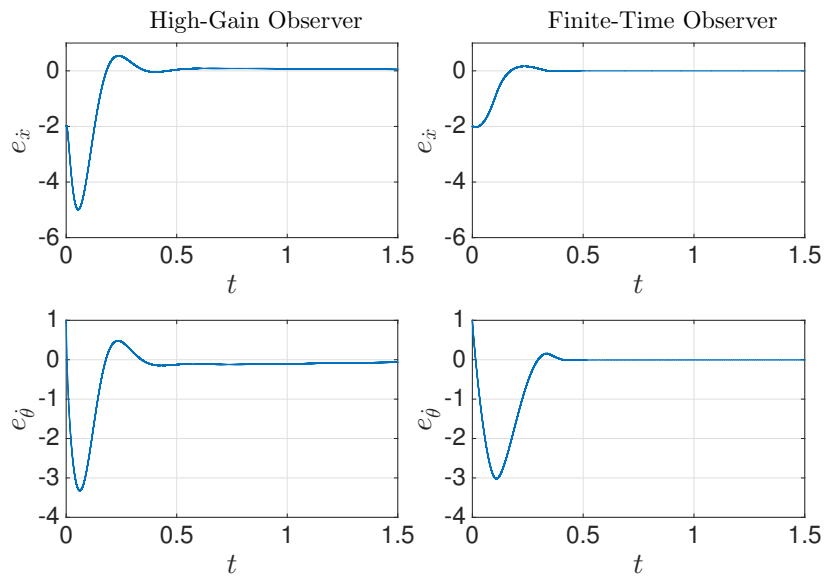

Fig. 1. Simulation plots of $e_{\dot{x}}$ and $e_{\dot{\theta}}$ for the HG and FT observers applied to the nonlinear plant with IC $x(0)=\left(0,-2, \frac{\pi}{4}, 1\right)$ and $K_{p}=10$.

ficiently large since the HG observer becomes more sensitive with respect to measurement noises as $\epsilon$ decreases. The estimation error of the FT observer turned out to be 10 times less $^{1}(\|e(t)\| \leq 0.01)$ for $t \geq 0.5 \mathrm{~s}$.

Figure 1 depicts the evolution of the observation errors of FT and HG observers for the noise-free case. Although the observers were designed using a linearized model, the system remains stable for the complete nonlinear model. It is also worth noting that the FT observer demonstrates a smaller peaking during transients.

To compare the observers for the case of noisy measurements, a band-limited white noise of power $10^{-5}$ has been added to the output signal during the simulation. The corresponding results are presented at Figure 2. They show almost twice better precision (in both $L_{(0.5,1.5)}^{2}$ and $L_{(0.5,1.5)}^{\infty}$ norms of the error) of the FT observer with respect to the HG one. This fact has a simple explanation in the context of high-gain observer theory: since the gain factor $\epsilon$ of the FT observer depends on the available part of the observation error (see Remark 12), namely,

$$
\epsilon=\epsilon(\sigma)=\left\|\tilde{P} C_{0}^{-1} \sigma\right\|^{\mu /(1+(m-1) \mu)}, \sigma=y-C \hat{x}=C e,
$$

then its value is automatically adapted to the noises of different magnitude (the larger the noise magnitude, the smaller the gain). In the noisy case the convergence time of the FT observer slightly increases, allowing a better estimation precision.

Example 2 (Uniformity w.r.t. to initial conditions). Here we compare the FT and the FX observers assuming that the FT observer is derived as the homogeneous approximation of the FX observer at zero, i.e. $L_{F T}=0.5 L_{F X}$ and $\tilde{P}=\tilde{P}_{1}$, where

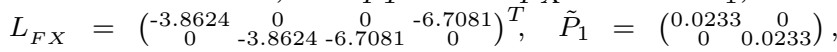
1 The numerical simulations have been done using the explicit
Euler method with sampling period $10^{-4}$. 

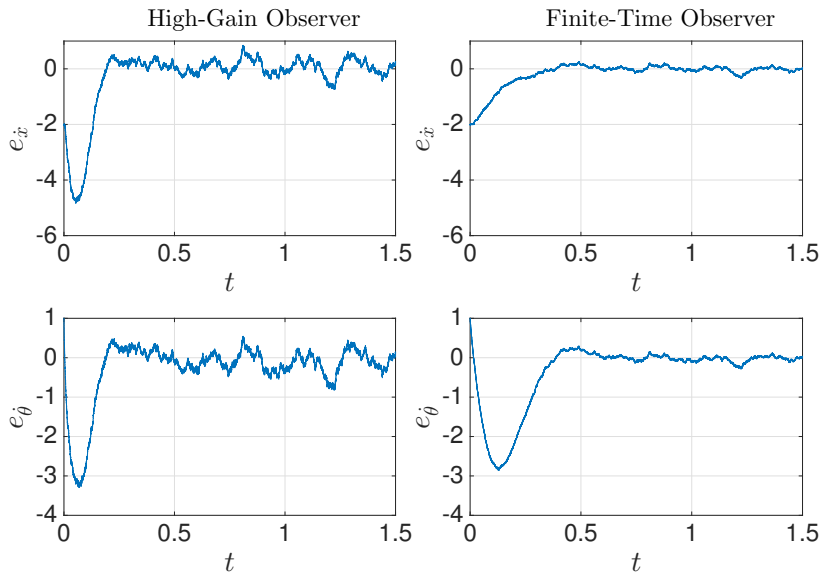

Fig. 2. Simulation plots of $e_{\dot{x}}$ and $e_{\dot{\theta}}$ for the HG and FT observers with a band-limited white noise in the measurements.

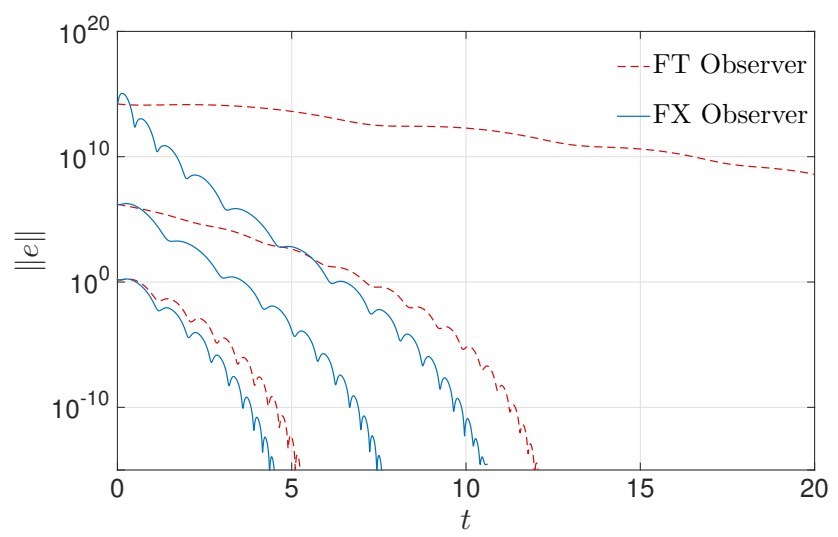

Fig. 3. Simulation plot of $\|e\|$ for the FT and FX observers applied to the linearized plant for three different IC $x(0)=\left(0,1, \frac{\pi}{4}, 0\right), x(0)=10^{3}\left(0,1, \frac{\pi}{4}, 0\right), x(0)=10^{7}\left(0,1, \frac{\pi}{4}, 0\right)$ and $K_{p}=5$.

$\tilde{P}_{2}=\left(\begin{array}{cc}0.2589 & 0 \\ 0 & 0.2589\end{array}\right)$ are the gain matrices of the FX observer obtained applying Algorithm 2 with the parameters $\left(\mu, \alpha, \tau, \xi, \delta_{1}, \delta_{2}, \beta\right)=(0.12,0.006,15,1,0.3,10 / 3,0.3)$.

The comparison results between the FT and FX observers in the noise-free case are depicted in Figure 3. They confirm low convergence time sensitivity with respect to initial conditions for the FX algorithm; for the FT one it is possible to see that the convergence time increases drastically as the norm of the initial conditions increases. The result of simulations are depicted using the logarithmic scale in order to demonstrate fast (hyper-exponential) convergence rate of the observers. Since locally (close to the origin of the error system) the FX algorithm almost coincides with the FT one, it has almost the same sensitivity with respect to measurement noises (see Fig. 4).

\section{Conclusion}

This article presents finite-time and fixed-time nonlinear observers for MIMO linear systems. Their key features are homogeneity properties and the use of the implicit Lyapunov function method for stability analysis of the error equation. The former allows the observers to attain non-asymptotic
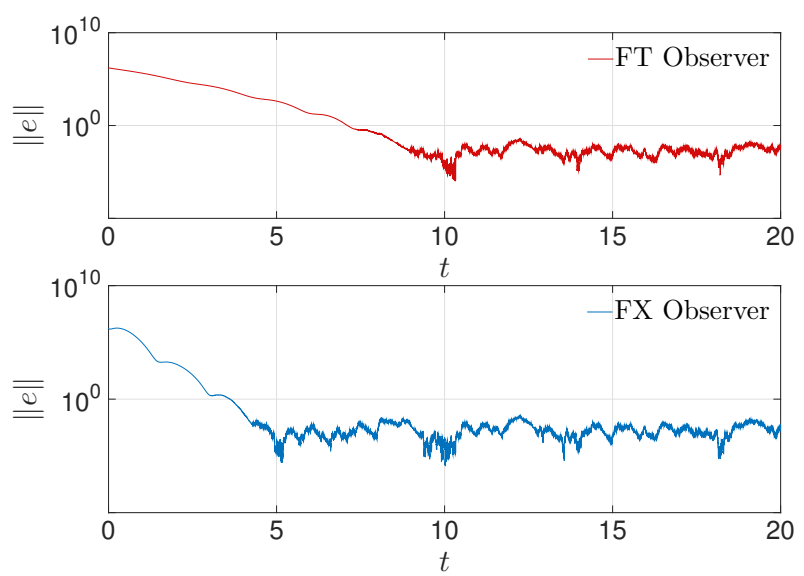

Fig. 4. Simulation plot of $\|e\|$ for the FT and FX observers with measurement band limited noise to the linearized plant with IC $x(0)=10^{3}\left(0,1, \frac{\pi}{4}, 0\right)$ and $K_{p}=5$.

(finite-time or fixed-time) convergence while the latter simplifies tuning of the observers' gains using LMI-based algorithms. The design is based on a transformation to a canonical observability form so that similar observers can be easily applied to nonlinear systems that admit this canonical form. The observers' robustness against bounded measurement noises and disturbances was also studied. It was shown that while both observers are ISS stable. Quantitative robustness analysis (e.g. construction of a sharp estimate of the observation error in the perturbed case) is considered as an important problem for further research.

\section{Acknowledgements}

This work was partially supported by the project Finite4SoS (ANR-15-CE23-0007).

\section{Appendix}

\subsection{Block Decomposition}

Let the matrices $T_{i}$ be defined by the following algorithm: Initialization : $A_{1}=A, C_{1}=C, T_{1}=I_{n}, m=1$.

Loop: While $\operatorname{rank}\left(C_{m}\right)<\operatorname{rown}\left(A_{m}\right)$ do $T_{m+1}=\left(\hat{C}_{m} C_{m}^{\perp}\right)$, $A_{m+1}=\left(C_{m}^{\perp}\right)^{T} A_{m} C_{m}^{\perp}, C_{m+1}=\hat{C}_{m}^{T} A_{m} C_{m}^{\perp}, m=m+1$, where $C_{m}^{\perp}:=\operatorname{null}\left(C_{m}\right), \hat{C}_{m}:=\operatorname{null}\left(\left(C_{m}^{\perp}\right)^{T}\right)$.

This simple algorithm can be easily realized in MATLAB and it helps to construct an orthogonal coordinate transformation that decomposes the original system (1) into a block upper diagonal canonical form. If the pair $(A, C)$ is observable, then the algorithm given above stops after $m$ steps, where $m<n$, and the ma$\operatorname{trix} \mathcal{O}=T_{1}\left(\begin{array}{cc}I_{w_{2}} & 0 \\ 0 & T_{2}\end{array}\right)\left(\begin{array}{cc}I_{w_{3}} & 0 \\ 0 & T_{3}\end{array}\right) \ldots\left(\begin{array}{cc}I_{w_{m}} & 0 \\ 0 & T_{m}\end{array}\right)$, where $w_{i}:=n-\operatorname{rown}\left(T_{i}\right)$, is an orthogonal matrix such that $\mathcal{O}^{T} \mathcal{O}=\mathcal{O O}^{T}=I_{n}$ and

$\mathcal{O} A \mathcal{O}^{T}=\left(\begin{array}{ccccc}A_{11} & A_{12} & 0 & \ldots & 0 \\ A_{21} & A_{22} & A_{23} & \ldots & 0 \\ \vdots & \vdots & \vdots & \ddots & \vdots \\ A_{m-11} & A_{m-12} & \ldots & A_{m-1} & A_{m-1 m} \\ A_{m 1} & A_{m 2} & \ldots & A_{m m-1} & A_{m m}\end{array}\right), C \mathcal{O}=\left(\begin{array}{llll}C_{0} & 0 & \ldots & 0\end{array}\right)$, 
where $C_{0}=C \hat{C}_{1}, A_{i j} \in \mathbb{R}^{n_{i} \times n_{j}}, n_{i}:=\operatorname{rank}\left(C_{i}\right), i, j=$ $1, \ldots, m$ and $\operatorname{rank}\left(A_{i i+1}\right)=n_{i+1}$. This can be proven, for example, using ideas of duality and Lemma 3 from [23].

Since $\operatorname{rank}\left(A_{i i+1}\right)=n_{i+1}=\operatorname{rown}\left(A_{i i+1}^{T}\right)$ then $A_{i i+1}^{T}$. $A_{i i+1}$ is invertible and $A_{i+1}^{+}=\left(A_{i+1}^{T} A_{i i+1}\right)^{-1} A_{i i+1}^{T}$ is the left inverse matrix to $A_{i+1}$. Consider now the next recursive algorithm in the matrix $\mathrm{A}$.

Initialization: $A_{i j}^{[m]}=A_{i j}, \quad i, j=1,2, . ., m$

Loop: for $q=m, m-1, \ldots, 2$

for $p=0,1, \ldots, q-2$

for $j=1,2, \ldots, q-p-1$

$A_{q j}^{[q-p-1]}=A_{q j}^{[q-p]}-A_{q q-p}^{[q-p]} \cdot A_{q-p-1 q-p}^{+} \cdot A_{q-p-1 j}^{[q-p]}$

end

$A_{q-1 q-p-1}^{[q-1]}=A_{q-1 q-p-1}^{[q]}+A_{q-1 q}^{[q]} \cdot A_{q q-p}^{[q-p]} \cdot A_{q-p-1 q-p}^{+}$

end

where the superscript $[m]$ represents the $m$ iteration over the matrix $A$. Then it can be shown that the transformation

$$
\Phi=\left(\begin{array}{ccccc}
I_{n_{1}} & 0 & \cdots & 0 & 0 \\
-A_{22}^{[2]} A_{12}^{+} & I_{n_{2}} & \cdots & 0 & 0 \\
\vdots & \vdots & \ddots & \vdots & \vdots \\
-A_{m-12}^{[2]} A_{12}^{+} & -A_{m-13}^{[3]} A_{23}^{+} & \cdots & I_{n_{m-1}} & 0 \\
-A_{m 2}^{[2]} A_{12}^{+} & -A_{m}^{[3]} A_{23}^{+} & \cdots & -A_{m m}^{[m]} A_{m-1}^{+} & I_{n_{m}}
\end{array}\right) \mathcal{O}
$$

reduces the original matrix $A$ to the block form: $\Phi A \Phi^{-1}=$ $F \tilde{C}+\tilde{A}$, where $n_{1}=k, F=\left(A_{11}^{[1]}, A_{21}^{[1]}, \ldots, A_{m-1}^{[1]}, A_{m 1}^{[1]}\right)^{T}$ and

$$
\tilde{C}=\left[\begin{array}{ll}
I_{n_{1}} & 0
\end{array}\right] \in \mathbb{R}^{n_{1} \times n}, \quad \tilde{A}=\left(\begin{array}{ccccc}
0 & A_{12} & 0 & \ldots & 0 \\
0 & 0 & A_{23} & \ldots & 0 \\
\vdots & \vdots & \vdots & \ddots & \vdots \\
0 & 0 & 0 & \ldots & A_{m-1 m} \\
0 & 0 & 0 & \ldots & 0
\end{array}\right) .
$$

\subsection{Proof of Theorem 13}

I. Show that the function $Q$ defined by (3) satisfies the conditions C1-C3 of Theorem 3. It is continuously differentiable on $\mathbb{R}_{+} \times \mathbb{R}^{n} \backslash\{0\}$. Since $P>0$ then the inequalities

$\frac{\lambda_{\min }(P)\|z\|^{2}}{\max \left\{V^{\left.2 \min r_{i}, V^{2} \max r_{i}\right\}}\right.} \leq Q(V, z)+1 \leq \frac{\lambda_{\max }(P)\|z\|^{2}}{\min \left\{V^{\left.2 \min r_{i}, V^{2} \max r_{i}\right\}}\right.}$ imply that for any $z \in \mathbb{R}^{n} \backslash\{0\}$ there exist $V^{-} \in \mathbb{R}_{+}$ and $V^{+} \in \mathbb{R}_{+}: Q\left(V^{-}, z\right)<0<Q\left(V^{+}, z\right)$. Moreover, if $Q(V, z)=0$ then, obviously, the condition $\mathbf{C 3}$ of Theorem 3 holds. Since $\frac{\partial Q}{\partial V}=-V^{-1} z^{T} D_{r}\left(V^{-1}\right)\left(H_{r} P+\right.$ $\left.P H_{r}\right) D_{r}\left(V^{-1}\right) z$ then $H_{r} P+P H_{r}>0$ implies $\frac{\partial Q}{\partial V}<0$ for $\forall V \in \mathbb{R}_{+}$and $z \in \mathbb{R}^{n} \backslash\{0\}$. So the condition $\mathbf{C 4}$ of Theorem 3 also holds. Therefore, the equation $Q(V, z)=0$ implicitly defines a positive definite Lyapunov function candidate $V: \mathbb{R}^{n} \rightarrow \mathbb{R}$

II. Let us denote $\lambda=\|\tilde{P} \tilde{C} e\| / V$ and show that $0 \leq \lambda \leq 1$ if (10d) holds. Let us denote $\tilde{e}=D_{r}\left(V^{-1}\right) e$. Hence, $\lambda=\|\tilde{P} \tilde{C} e\| / V=\|\tilde{P} \tilde{C} \tilde{e}\|$ and $\tilde{e}^{T} P \tilde{e}=1$ (due to $Q(V, e)=0$ ). Given (10d) and that $\tilde{P}=X^{1 / 2}$, we have $\lambda^{2}=\tilde{e}^{T} \tilde{C}^{T} X \tilde{C} \tilde{e} \leq \tilde{e}^{T} P \tilde{e}=1$.

III. Denote by $\partial Q^{e}$ the partial derivative of $Q$ along (9): $\partial Q^{e}=2 e^{T} D_{r}\left(V^{-1}\right) P D_{r}\left(V^{-1}\right)\left(\tilde{A}+D_{\tilde{r}}\left(\|\tilde{P} \tilde{C} e\|^{-1}\right) L_{F T} \tilde{C}\right) e$.
Taking into account the identities $D_{\tilde{r}}(V) V^{-1}=D_{r}\left(V^{-1}\right)$. $V^{\mu /(1+(m-1) \mu)}, \quad D_{r}\left(V^{-1}\right) \tilde{A} D_{r}^{-1}\left(V^{-1}\right)=V^{\frac{-\mu}{1+(m-1) \mu}} \tilde{A} \quad$ and $D_{\tilde{r}}\left(V^{-1}\right) L_{F T} \tilde{C}=V^{\frac{-\mu}{1+(m-1) \mu}} D_{r}^{-1}\left(V^{-1}\right) L_{F T} \tilde{C} D_{r}\left(V^{-1}\right)$ we derive

$\partial Q^{e}=V^{\frac{-\mu}{1+(m-1) \mu}}\left(\begin{array}{c}D_{r}\left(V^{-1}\right) e \\ \Xi(\lambda) L_{F T} \frac{\tilde{\tilde{\tilde{C}} e}}{\| \tilde{P} \tilde{C} e}\end{array}\right)^{T} \Upsilon\left(\begin{array}{c}D_{r}\left(V^{-1}\right) e \\ \Xi(\lambda) L_{F T} \frac{\tilde{\tilde{C} e}}{\|\tilde{P} \tilde{C} e\|}\end{array}\right)$ where $\Upsilon=\left(\begin{array}{crr}P\left(\tilde{A}+L_{F T} \tilde{C}\right)+\left(\tilde{A}+L_{F T} \tilde{C}\right)^{T} & P & P \\ P\end{array}\right)$. Using the matrix inequality (10a) with $Y=P L_{F T}$ and the identity $e^{T} D_{r}\left(V^{-1}\right) P D_{r}\left(V^{-1}\right) e=1$ we estimate

$$
\begin{gathered}
\partial Q^{e} \leq V^{\frac{-\mu}{1+(m-1) \mu}}\left(-\alpha e^{T} D_{r}\left(V^{-1}\right)\left(P H_{r}+H_{r} P\right) D_{r}\left(V^{-1}\right) e+\right. \\
\left.\xi \frac{(\tilde{P} \tilde{C} e)^{T}}{\|\tilde{P} \tilde{C} e\|} \tilde{P}^{-1} L_{F T}^{T} \Xi(\lambda) Z \Xi(\lambda) L_{F T} \tilde{P}^{-1} \frac{\tilde{P} \tilde{C} e}{\|\tilde{P} \tilde{C} e\|}-\xi\right) .
\end{gathered}
$$

Since (10c) is equivalent to $\tilde{P}^{-1} L_{F T}^{T} P L_{F T} \tilde{P}^{-1} \leq \tau I_{k}$ with $\tilde{P}=X^{1 / 2}$ and taking into account (10d) and (10f) we derive $\partial Q_{e} \leq \frac{-\alpha e^{T} D_{r}\left(V^{-1}\right)\left(P H_{r}+H_{r} P\right) D_{r}\left(V^{-1}\right) e}{V^{\frac{\mu}{1+(m-1) \mu}}}<\alpha V^{1-\frac{\mu}{1+(m-1) \mu}} \frac{\partial Q}{\partial V}$. Finally, applying Theorem 3 we finish the proof.

\subsection{Proof of Proposition 15}

Denote $W(\lambda)=z^{T} \Xi(\lambda) Z \Xi(\lambda) z$. Since $\Xi^{\prime}(\theta)=\frac{1}{\theta} \Xi(\theta)\left(I_{n}-\right.$ $\left.H_{\tilde{r}}\right)-H_{\tilde{r}}$, then $W^{\prime}(\theta)=z^{T}\left(\Xi^{\prime}(\theta) Z \Xi(\theta)+\Xi(\theta) Z \Xi^{\prime}(\theta)\right) z=$

$$
z^{T}\left(\begin{array}{c}
\Xi(\theta) \\
I_{n}
\end{array}\right)^{T}\left(\begin{array}{cc}
\left(\frac{1}{\theta}\left(I_{n}-H_{\tilde{r}}\right) Z+\frac{1}{\theta} Z\left(I_{n}-H_{\tilde{r}}\right)-Z H_{\tilde{r}}\right. \\
-H_{\tilde{r}} Z
\end{array}\right)\left(\begin{array}{c}
\Xi(\theta) \\
I_{n}
\end{array}\right) z-\theta z^{T} M z
$$

and due to (11c) and the Schur complement we have $W^{\prime}(\theta) \geq-\theta z^{T} M z$ and $W(\lambda) \leq W\left(q_{i}\right)-\frac{1}{2}\left(\lambda^{2}-q_{i}^{2}\right) z^{T} M z$ for any $\lambda \in\left[q_{i-1}, q_{i}\right], i=1, \ldots ., N$. Hence, the set of inequalities (11) imply (10f).

\subsection{Proof of Theorem 16}

I. The function $Q_{i}$ defined by (3) with $r=r_{i}, i=1,2$ satisfies the conditions C1-C4 of Theorem 3 (see proof of Theorem 13). Note that $Q_{1}(1, z)=Q_{2}(1, z)$ for all $z \in \mathbb{R}^{n}$. In order to complete the proof we need to show that the conditions C7-C8 of Theorem 4 hold.

II. Let $\tilde{P}_{i}=\delta_{i} \tilde{P}$ with $\tilde{P}=X^{1 / 2}$. In Subsection 8.2, it was shown that $0 \leq \lambda_{1}=\frac{\|\tilde{P} \tilde{C} e\|}{V} \leq 1$ with $Q(V, e)=0$ if $(14 \mathrm{e})$ holds. The same result remains true for $Q_{i}(V, e)=0$.

III. Let $\partial Q_{i}^{e}$ be the derivative of $Q_{i}$ along the equation (13) $\partial Q_{i}^{e}=2 e^{T} D_{r_{i}}\left(V^{-1}\right) P D_{r_{i}}\left(V^{-1}\right)\left(\tilde{A}+\frac{D_{\tilde{r}}\left(\frac{1}{\delta_{1} \lambda_{1} V}\right)+D_{\tilde{r}}\left(\delta_{2} \lambda_{1} V\right)}{2} L_{F X} \tilde{C}\right) e$, $\lambda_{1}=\frac{\|\tilde{P} \tilde{C} e\|}{V}$. Since $D_{r_{i}}(\rho)=\rho^{1+\frac{(-1)^{i+1} \mu}{1+(m-1) \mu}} D_{\tilde{r}}\left(\rho^{(-1)^{i}}\right), \rho>0$ then $\partial Q_{i}^{e}=V^{\frac{(-1)^{i} \mu}{1+(m-1) \mu}}\left(\begin{array}{c}D_{r_{i}}\left(V^{-1}\right) e \\ \bar{\Xi}_{i}^{\delta}(\lambda) L_{F X} \frac{\tilde{C} e}{\|\tilde{P} \tilde{C} e\|}\end{array}\right)^{T} \chi\left(\begin{array}{c}D_{r_{i}}\left(V^{-1}\right) e \\ \bar{\Xi}_{i}^{\delta}(\lambda) L_{F X} \frac{\tilde{C} e}{\|\tilde{P} \tilde{C} e\|}\end{array}\right)$,

$\lambda=\left(\lambda_{1}, \lambda_{2}\right)$, where $\chi=\left(\begin{array}{cr}P\left(\tilde{A}+L_{F X} \tilde{C}\right)+\left(\tilde{A}+L_{F X} \tilde{C}\right)^{T} P & P \\ P\end{array}\right)$, $\lambda_{2}=V^{2} \in(0,1]$ if $i=1$ and $\lambda_{2}=1 / V^{2} \in(0,1]$ if $i=2$. Repeating the considerations of the proof of Theorem 13 we derive that (14) imply $\partial Q_{i}^{e} \leq$ $-\alpha V^{\frac{(-1)^{i} \mu}{1+(m-1) \mu}} e^{T} D_{r_{i}}\left(\frac{1}{V}\right)\left(P H_{i}+H_{i} P\right) D_{r_{i}}\left(\frac{1}{V}\right) e$. Taking into account $\frac{\partial Q_{i}(V, e)}{\partial V}=-V^{-1} e^{T} D_{r_{i}}\left(V^{-1}\right)\left(H_{i} P+P H_{i}\right) D_{r_{i}}\left(V^{-1}\right) e$ and LMI (14d) we derive that all conditions of Theorem 4 hold, so the error equation (13) is fixed-time stable and $T_{\max } \leq \frac{1+(m-1) \mu}{0.5 \alpha \mu}$. 


\subsection{Proof of Proposition 17}

I. Consider the function $W_{i}(\lambda)=z^{T} \bar{\Xi}_{i}^{\delta}(\lambda) Z_{i} \bar{\Xi}_{i}^{\delta}(\lambda) z$, where $z \in \mathbb{R}^{n}$ is an arbitrary non-trivial vector. Since $\frac{\partial \bar{\Xi}_{i}^{\delta}}{\partial \lambda_{1}}=$ $\frac{1}{\lambda_{1}} \bar{\Xi}_{i}^{\delta}(\lambda)+\frac{1}{2}\left(D_{\tilde{r}}\left(\delta_{2} \lambda_{1} / \lambda_{2}^{i-2}\right)-D_{\tilde{r}}\left(\lambda_{2}^{i-1} /\left(\delta_{1} \lambda_{1}\right)\right)\right) H_{\tilde{r}}=$ $\frac{1}{\lambda_{1}}\left(\bar{\Xi}_{i}^{\delta}(\lambda)\left(I_{n}-H_{\tilde{r}}\right)+D_{\tilde{r}}\left(\delta_{2} \lambda_{1} / \lambda_{2}^{i-2}\right) H_{\tilde{r}}+H_{\tilde{r}}\right)$ then

$$
\begin{aligned}
& \frac{\partial W_{i}}{\partial \lambda_{1}}=z^{T} \frac{\partial \bar{\Xi}_{i}}{\partial \lambda_{1}} Z \bar{\Xi}_{i}^{\delta} z+z^{T} \bar{\Xi}_{i}^{\delta} Z \frac{\partial \bar{\Xi}_{i}^{\delta}}{\partial \lambda_{1}} z=
\end{aligned}
$$

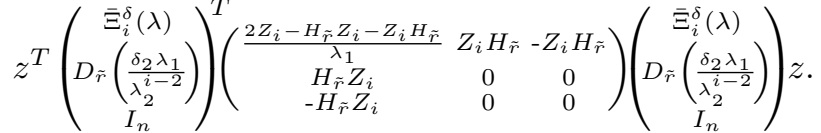

Using the inequality (15c) we derive $\frac{\partial W_{i}}{\partial \lambda_{1}} \geq-\lambda_{1} \Psi_{i}(\lambda)$ $-\lambda_{1} z^{T} S_{i} z$, where $\Psi_{i}(\lambda)=z^{T} D_{\tilde{r}}\left(\frac{\delta_{2} \lambda_{1}}{\lambda_{2}^{i-2}}\right) M_{i} D_{\tilde{r}}\left(\frac{\delta_{2} \lambda_{1}}{\lambda_{2}^{i-2}}\right) z$. On the other hand, the inequality $M_{i} H_{\tilde{r}}+H_{\tilde{r}} M_{i}>0$ implies the estimates $\frac{\partial \Psi_{i}(\lambda)}{\partial \lambda_{1}}>0, \frac{\partial \Psi_{i}(\lambda)}{\partial \lambda_{2}} \geq 0$. Hence we conclude $\frac{\partial W_{i}}{\partial \lambda_{1}} \geq-\lambda_{1} z^{T} D_{\tilde{r}}\left(\delta_{2}\right) M_{i} D_{\tilde{r}}\left(\delta_{2}\right) z-\lambda_{1} z^{T} S_{i} z$ for $\lambda \in[0,1] \times[0,1]$ and $W_{i}(\lambda) \leq W_{i}\left(q_{j}, \lambda_{2}\right)+$ $\frac{q_{j}^{2}-\lambda_{1}^{2}}{2} z^{T}\left(D_{\tilde{r}}\left(\delta_{2}\right) M_{i} D_{\tilde{r}}\left(\delta_{2}\right)+S_{i}\right) z$ for all $\lambda_{1} \in\left[q_{j-1}, q_{j}\right]$.

II. Since $\bar{\Xi}_{i}^{\delta}(\lambda)=\bar{\Xi}_{i}^{\delta}(\tilde{\lambda})+\frac{\lambda_{1}}{2} D_{\tilde{r}}\left(\lambda_{1}^{(-1)^{i+1}}\right) D_{\tilde{r}}\left(\lambda_{2}\right)$ with $\tilde{\lambda}=$ $\left(\lambda_{1}, 0\right)$, then we derive the identity $W_{i}(\lambda)=z^{T} B_{i}\left(\lambda_{1}\right)^{T}$ $F_{i}\left(\lambda_{2}\right) B_{i}\left(\lambda_{1}\right) z$, where $F_{i}\left(\lambda_{2}\right)=\left(\begin{array}{c}D_{\tilde{r}}\left(\lambda_{2}\right) \\ I_{n}\end{array}\right) Z_{i}\left(\begin{array}{c}D_{\tilde{r}}\left(\lambda_{2}\right) \\ I_{n}\end{array}\right)^{T}$ and $B_{i}\left(\lambda_{1}\right)=\left(\begin{array}{c}\frac{\lambda_{1}}{2} D_{\tilde{r}}\left(\left(\lambda_{1} \delta_{3-i}\right)^{(-1)^{i+1}}\right) \\ \Xi_{i}^{\delta}(\tilde{\lambda})\end{array}\right)$. Let us denote $\kappa\left(\lambda_{2}\right)=\ln \left(\lambda_{2} W\right)$. Since $\kappa$ has the derivative $\frac{d \kappa}{d \lambda_{2}}=$ $\frac{1}{\lambda_{2} W}\left(W+z^{T} B_{i}\left(q_{j}\right)^{T}\left(\begin{array}{cc}D_{\tilde{r}}\left(\lambda_{2}\right) & 0 \\ 0 & I_{n}\end{array}\right) \Gamma_{i}\left(\begin{array}{cc}D_{\tilde{r}}\left(\lambda_{2}\right) & 0 \\ 0 & I_{n}\end{array}\right) B_{i}\left(q_{j}\right) z\right)$, where $\Gamma_{i}=\left(\begin{array}{cc}H_{\tilde{r}} Z_{i}+Z_{i} H_{\tilde{r}} & H_{\tilde{r}} Z_{i} \\ Z_{i} H_{\tilde{r}} & 0\end{array}\right)$. Since $\Gamma_{i}+\left(\begin{array}{cc}0 & 0 \\ 0 & U_{i}\end{array}\right) \geq 0$ due to (15a) then $\frac{d \kappa}{d \lambda_{2}} \geq \frac{1}{\lambda_{2}}-c e^{-\kappa\left(\lambda_{2}\right)}$ with $c=$ $z^{T} \bar{\Xi}_{i}^{\delta}(\tilde{\lambda}) U_{i} \bar{\Xi}_{i}^{\delta}(\tilde{\lambda}) z$. Hence $W(\lambda) \leq W\left(\lambda_{1}, p_{s}\right)+c \ln \left(p_{s} \lambda^{-1}\right)$ $\leq W\left(\lambda_{1}, p_{s}\right)+c \ln \left(\frac{p_{s}}{p_{s-1}}\right)$ for all $\lambda_{2} \in\left[p_{s-1}, p_{s}\right]$. Therefore, LMIs (15a-15d) imply $W(\lambda) \leq \tau^{-1} z^{T} P z$ for all $\lambda \in[0,1] \times\left[p_{0}, 1\right]$. Finally, it is easy to check that $W_{i}(\lambda) \leq$ $\tilde{W}(\lambda):=z^{T} B_{i}\left(\lambda_{1}\right)^{T}\left(\begin{array}{cc}D_{\tilde{r}}\left(\lambda_{2}\right) Z_{i} D_{\tilde{r}}\left(\lambda_{2}\right)+D_{\tilde{r}}\left(\lambda_{2}\right) & 0 \\ 0 & Z_{i}+Z_{i} D_{\tilde{r}}\left(\lambda_{2}\right) Z_{i}\end{array}\right)$ $B_{i}\left(\lambda_{1}\right) z$. Since $Z_{i} H_{\tilde{r}}+H_{\tilde{r}} Z_{i} \geq 0$ then $\frac{\partial \tilde{W}}{\partial \lambda_{2}} \geq 0$ and $W(\lambda) \leq \tilde{W}\left(\lambda_{1}, p_{0}\right)$ for all $\lambda_{2} \in\left[0, p_{0}\right]$. Therefore, the inequality (15e) implies $W(\lambda) \leq \tau^{-1} z^{T} P z$ for all $\lambda \in[0,1] \times\left[0, p_{0}\right]$.

\subsection{Proof of Corollary 18}

Denote $\tilde{f}(e, d)$ the right-hand side of (16), where $d=$ $\left(d_{x}, d_{y}\right)$. For $d=0$ it coincides with the right-hand side of (9) and defines an $r$-homogeneous vector field with degree $\frac{-\mu}{1+(m-1) \mu}<0$. Taking into account that $D_{r}^{-1}(\lambda) \tilde{A} D_{r}(\lambda)=\lambda^{-\frac{\mu}{1+(m-1) \mu}} \tilde{A}$ and $\tilde{C} D_{r}(\lambda) e=\lambda^{r_{1}} \tilde{C} e$ we derive $\lambda^{\frac{\mu}{1+(m-1) \mu}} D_{r}^{-1}(\lambda)\left(\tilde{A}+D_{\tilde{r}}\left(\frac{1}{\left\|\tilde{P} \tilde{C} D_{r}(\lambda) e\right\|}\right) L_{F T} \tilde{C}\right) D_{r}(\lambda) e$ $=\left(\tilde{A}+D_{\tilde{r}}\left(\|\tilde{P} \tilde{C} e\|^{-1}\right) L_{F T} \tilde{C}\right) e$, therefore the error dynamics (9) is $r$-homogeneous of degree $\eta=-\frac{\mu}{1+(m-1) \mu}$. Selecting $\bar{r}=\left(\mathbb{1}_{k}, r-\frac{\mu}{1+(m-1) \mu} \mathbb{1}_{n}\right) \in \mathbb{R}^{k+n}$ and using Theorem 9 , we conclude ISS for system (16) for $\mu \in(0,1)$. If $\mu=1$ then $\bar{r}_{\min }=0$ and only iISS can be asserted for (16).

\subsection{Proof of Corollary 19}

Denote $\tilde{f}(e, d)$ the right-hand side of (17), where $d=$ $\left(d_{x}, d_{y}\right)$. For $d=0$ it defines a vector field $f(\cdot)=\tilde{f}(\cdot, 0)$ that is locally homogeneous at 0 and at $+\infty$, namely, $\left(r_{1}, 0, f_{0}\right)$-homogeneous with negative degree $\eta_{0}=$ $-\frac{\mu}{1+(m-1) \mu}$, and $\left(r_{2},+\infty, f_{\infty}\right)$-homogeneous with positive degree $\eta_{\infty}=\frac{\mu}{1+(m-1) \mu}, f_{0}=\left(\tilde{A}+\frac{1}{2} D_{\tilde{r}}\left(\left\|\tilde{P}_{1} \tilde{C} e\right\|^{-1}\right) L_{F X} \tilde{C}\right) e$ and $f_{\infty}=\left(\tilde{A}+\frac{1}{2} D_{\tilde{r}}\left(\left\|\tilde{P}_{2} \tilde{C} e\right\|\right) L_{F X} \tilde{C}\right) e$.

Indeed, $\lim _{\lambda_{0} \rightarrow 0}\left[\lambda^{-\eta} D_{r_{1}}^{-1}(\lambda)\left[\frac{D_{\tilde{r}}\left(\left\|\tilde{P}_{1} \tilde{C} D_{r_{1}}(\lambda) e\right\|^{-1}\right)+D_{\tilde{r}}\left(\left\|\tilde{P}_{2} \tilde{C} D_{r_{1}}(\lambda) e\right\|\right)}{2}\right.\right.$ $\left.L_{F X} \tilde{C} D_{r_{1}}(\lambda) e+\tilde{A} D_{r_{1}}(\lambda) e\right]-\left(A e+\frac{1}{2} D_{\tilde{r}}\left(\left\|\tilde{P}_{1} \tilde{C} e\right\|^{-1}\right)\right.$ $\left.\left.L_{F X} \tilde{C} e\right)\right]=0$ for $\eta=-\frac{\mu}{1+(m-1) \mu}<0$, so the $r_{1}$ homogeneous approximation of degree $\eta$ around 0 of (13) is $f_{0}$. Analogously it can be shown that $f_{\infty}$ is the $r_{2}$ homogeneous approximation of (13) with degree $\eta_{\infty}=$ $\frac{\mu}{1+(m-1) \mu}>0$ at $+\infty$. It is worth stressing that if all the conditions of Theorem 16 hold, then the origins of $\dot{e}=f_{0}(e)$, $\dot{e}=f(e)$ and $\dot{e}=f_{\infty}(e)$ are globally asymptotically stable. Hence, selecting $\bar{r}^{0}=\left(\mathbb{1}_{k}, r_{1}-\frac{\mu}{1+(m-1) \mu} \mathbb{1}_{n}\right) \in \mathbb{R}^{k+n}$ and $\bar{r}^{\infty}=\left(\mathbb{1}_{k}, r_{2}+\frac{\mu}{1+(m-1) \mu} \mathbb{1}_{n}\right) \in \mathbb{R}^{k+n}$, and using Theorem 10 we derive that the system (17) is ISS.

\section{References}

[1] R. Kalman, "A new approach to linear filtering and prediction problems," Journal of Basic Engineering -, vol. 82, no. 1, pp. 3545, 1960.

[2] D. Luenberger, "Observing the state of a linear system," IEEE Transactions on Military Electronics, vol. 8, no. 2, pp. 74-80, 1964.

[3] V. Andrieu, L. Praly, and A. Astolfi, "High-gain observers with updated high-gain and homogeneous correction terms," Automatica, vol. 45, no. 2, pp. 422-428, 2009.

[4] M. Ghanes, J. De Leon, and J. P. Barbot, "Observer design for nonlinear systems under unknown time-varying delays," IEEE Transactions on Automatic Control, vol. 58, no. 6, pp. 1529-1534, 2013.

[5] F. Mazenc and O. Bernard, "Interval observers for linear timeinvariant systems with disturbances," Automatica, vol. 47, no. 1, pp. 140-147, 2011.

[6] A. Zolghadri, D. Henry, J. Cieslak, D. Efimov, and P. Goupil, Fault Diagnosis and Fault-Tolerant Control and Guidance for Aerospace Vehicles. Springer, 2014.

[7] W. Lahoz, B. Khattatov, and R. Menard, Eds., Data Assimiliation: Making Sense of Observations. Springer, 2010.

[8] H. Rios, D. Efimov, J. A. Moreno, W. Perruquetti, and J. G. Rueda-Escobedo, "Time-varying parameter identification algorithms: Finite and fixed-time convergence," IEEE Transactions on Automatic Control, 2017.

[9] R. Engel and G. Kreisselmeier, "A continuous-time observer which converges in finite time," IEEE Transactions on Automatic Control, vol. 47, pp. 1202-1204, 2002.

[10] V. Andrieu, L. Praly, and A. Astolfi, "Homogeneous approximation, recursive observer and output feedback," SIAM Journal of Control and Optimization, vol. 47(4), pp. 1814-1850, 2008.

[11] W. Perruquetti, T. Floquet, and E. Moulay, "Finite-time observers: Application to secure communication," IEEE Transactions on Automatic Control, vol. 53(1), pp. 356-360, 2008. 
[12] Y. Aoustin, C. Chevallereau, and Y. Orlov, "Finite time stabilization of a perturbed double integrator - part II: applications to bipedal locomotion," in Conference on Decision and Control, 2010, pp. 3554-3559.

[13] T. Floquet, C. Edwards, and S. Spurgeon, "On sliding mode observers for systems with unknown inputs," International Journal of Adaptive Control and Signal Processing, vol. 21, no. 8-9, pp. 638-656, 2007.

[14] H. K. Khalil and L. Praly, "High-gain observers in nonlinear feedback control," International Journal of Robust and Nonlinear Control, vol. 24, no. 6, pp. 993-1015, 2014.

[15] A. Levant, "Robust exact differentiation via sliding mode technique," Automatica, vol. 34, no. 3, pp. 379-384, 1998.

[16] E. Cruz-Zavala, J. Moreno, and L. Fridman, "Uniform robust exact differentiator," IEEE Transactions on Automatic Control, vol. 56, no. 11, pp. 2727-2733, 2011.

[17] M. Angulo, J. Moreno, and L. Fridman, "Robust exact uniformly convergent arbitrary order differentiator," Automatica, vol. 49, no. 8 , pp. 2489-2495, 2013.

[18] F. Lopez-Ramirez, A. Polyakov, D. Efimov, and W. Perruquetti, "Finite-Time and Fixed-Time Observers Design via Implicit Lyapunov Function," in European Control Conference 2016, Aalborg, Denmark, Jun. 2016.

[19] S. Boyd, E. Ghaoui, E. Feron, and V. Balakrishnan, Linear Matrix Inequalities in System and Control Theory. Philadelphia: SIAM, 1994.

[20] E. Roxin, "On finite stability in control systems," Rendiconti del Circolo Matematico di Palermo, vol. 15(3), pp. 273-283, 1966.

[21] S. Bhat and D. Bernstein, "Finite-time stability of continuous autonomous systems," SIAM Journal of Control and Optimization, vol. 38(3), pp. 751-766, 2000.

[22] Y. Orlov, "Finite time stability and robust control synthesis of uncertain switched systems," SIAM Journal of Control and Optimization, vol. 43(4), pp. 1253-1271, 2005.

[23] A. Polyakov, "Nonlinear feedback design for fixed-time stabilization of linear control systems," IEEE Transactions on Automatic Control, vol. 57, no. 8, pp. 2106-2110, 2012.

[24] A. Polyakov, D. Efimov, and W. Perruquetti, "Finite-time and fixedtime stabilization: Implicit Lyapunov function approach," Automatica, vol. 51, pp. 332-340, 2015.

[25] R. Courant and F. John, Introduction to calculus and analysis (Vol. II/1). New York: Springer, 2000.

[26] V. Korobov, "A general approach to synthesis problem," Doklady Academii Nauk SSSR, vol. 248, pp. 1051-1063, 1979.

[27] J. Adamy and A. Flemming, "Soft variable-structure controls: a survey," Automatica, vol. 40, pp. 1821-1844, 2004.

[28] A. Filippov, Differential equations with discontinuous right-hand sides. Kluwer, Dordrecht, 1988.

[29] A. Bacciotti and L. Rosier, Lyapunov Functions and Stability in Control Theory, 2nd ed., B. Springer, Ed. Springer, 2005.

[30] E. Bernuau, A. Polyakov, D. Efimov, and W. Perruquetti, "Verification of ISS, iISS and IOSS properties applying weighted homogeneity," Systems \& Control Letters, vol. 62, no. 12, pp. 1159-1167, 2013.

[31] H. Shim and D. Liberzon, "Nonlinear observers robust to measurement disturbances in an ISS sense," IEEE Transactions on Automatic Control, vol. 61, no. 1, pp. 48-61, 2016.

[32] S. Dashkovskiy, D. V. Efimov, and E. D. Sontag, "Input to state stability and allied system properties," Automation and Remote Control, vol. 72, no. 8, pp. 1579-1614, 2011.

[33] W. M. Wonham, Linear Multivariable Control: A Geometric Approach. Springer, 1985.
[34] S. V. Drakunov, D. B. Izosimov, A. G. Luk'yanov, V. A. Utkin, and V. I. Utkin, "The block control design principle I," Automation and Remote Control, vol. 51, no. 5, pp. 601-608, 1990.

[35] _ - "The block control design principle II," Automation and Remote Control, vol. 51, no. 6, pp. 737-746, 1990.

[36] M. Misrikhanov and V. Ryabchenko, "Pole placement for controlling a large scale power system," Automation and Remote Control, vol. 72, no. 10, pp. 2123-2146, 2011.

[37] A. Isidori, Nonlinear Control Systems. Springer-Verlag, N. Y. Inc., 1995.

[38] A. Prasov and H. Khalil, "A nonlinear high-gain observer for systems with measurement noise in a feedback control framework," IEEE Transactions on Automatic Control, vol. 58, no. 3, pp. 569-580, 2013.

[39] R. Sanfelice and L. Praly, "On the performance of high-gain observers with gain adaptation under measurement noise," Automatica, vol. 47, pp. 2165-2176, 2011.

[40] T. Menard, E. Moulay, and W. Perruquetti, "Fixed-time observer with simple gains for uncertain systems," Automatica, vol. 81, no. 7, pp. 438-446, 2017. 